INPLASY

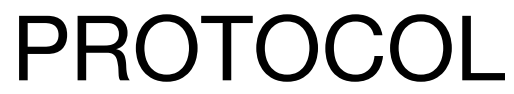

To cite: Qiao et al. Efficacy of total hip arthroplasty for the treatment of patients with femoral head avascular necrosis: a protocol for systematic review and metaanalysis. Inplasy protocol 202040067. doi: 10.37766/inplasy2020.4.0067

Received: 12 April 2020

Published: 12 April 2020

Corresponding author: Qing-hui Ji

qinghui9652@yeah.net

Author Affiliation:

First Affiliated Hospital of Jiamusi University

Support: HLJPUBRBERP (2017-KYYWF-0574)

Review Stage at time of this submission: The review has not yet started.

Conflicts of interest: None.

\section{Efficacy of total hip arthroplasty for the treatment of patients with femoral head avascular necrosis: a protocol for systematic review and} meta-analysis

Qiao, XF1; Xue, Y2; Liu, SC3; Ji, QH4.

Review question / Objective: Does total hip arthroplasty (THAP) effectively treat femoral head avascular necrosis (FHAN)?

Condition being studied: Femoral head avascular necrosis; total hip arthroplasty.

Information sources: The following electronic bibliographic databases will be searched from inception to the February 29, 2020: MEDLINE, EMBASE, Cochrane Library, Cumulative Index to Nursing and Allied Health Literature, China National Knowledge Infrastructure, and Chinese Scientific Journal Database. We will identify any potential RCTs of THAP for the treatment of patients with FHAN. We will search all these electronic databases without any language and publication status restrictions. The search strategy for MEDLINE is presented. We will modify similar search strategies to all other electronic databases. Other resources for potential studies are also searched, such as websites of clinical trial registry, and reference lists of included trials and relevant reviews.

INPLASY registration number: This protocol was registered with the International Platform of Registered Systematic Review and Meta-Analysis Protocols (INPLASY) on 12 April 2020 and was last updated on 12 April 2020 (registration number INPLASY202040067).

\section{INTRODUCTION}

Review question / Objective: Does total hip arthroplasty (THAP) effectively treat femoral head avascular necrosis (FHAN)?

Condition being studied: Femoral head avascular necrosis; total hip arthroplasty.

\section{METHODS}

Participant or population: Participants were diagnosed with FHAN will be included regardless their age, sex, and source of the studies. 
Intervention: Any types of THAP therapy used in patients as interventional intervention will be included in this study.

Comparator: We will include patients who received any management as a control intervention in this study. We will exclude any combined therapy with THAP.

Study designs to be included: Randomized controlled trials (RCTs) of THAP for the treatment of FHAN, which were reported in any language, will be included.

Eligibility criteria: This study will include RCTs that compared the efficacy and safety of THAP with other treatments for the treatment of patients with FHAN.

Information sources: The following electronic bibliographic databases will be searched from inception to the February 29, 2020: MEDLINE, EMBASE, Cochrane Library, Cumulative Index to Nursing and Allied Health Literature, China National Knowledge Infrastructure, and Chinese Scientific Journal Database. We will identify any potential RCTs of THAP for the treatment of patients with FHAN. We will search all these electronic databases without any language and publication status restrictions. The search strategy for MEDLINE is presented. We will modify similar search strategies to all other electronic databases. Other resources for potential studies are also searched, such as websites of clinical trial registry, and reference lists of included trials and relevant reviews.

Main outcome(s): Primary outcome includes pain intensity of hip or knee joints. It is measured by any validated pain scales, such as Numerical Rating Scale.

Additional outcome(s): Secondary outcomes are function and limitation of attacked femoral head (as checked by any relevant validated indexes, including Western Ontario and McMaster Universities Osteoarthritis Index), and health-related quality of life (as evaluated by any related tools, such as 36-Item Short Form Health
Survey), and any complications post surgery.

Data management: The following data will be extracted from all included RCTs by two independent authors using predefined data acquisition form: reference identification, author information, publication time, patient characteristics, sample size, study setting, study methods, details of intervention and controls, outcome indicators at all reported time points, complications, follow-up information, results, findings, and conflict of interests. Any different opinions will be arbitrated by a third author through discussion.

Quality assessment / Risk of bias analysis: Risk of bias for each eligible trial will be assessed by two independent authors using Cochrane Risk of Bias Tool. Two authors will independently judge them for each study on seven aspects. Each aspect will be categorized as high, unclear or low risk of bias. Any disagreements regarding the risk of bias assessment will be solved by a third author through discussion.

Strategy of data synthesis: RevMan $\mathbf{5 . 3}$ software will be used for data synthesis and statistically analysis. As for dichotomous outcome data (including incidence of complications), risk ratio and 95\% confidence intervals (Cls) will be employed. As for continuous outcome data (including pain intensity of hip or knee joints, function and limitation of attacked femoral head, and health-related quality of life), mean difference or standardized mean difference and $95 \% \mathrm{Cls}$ will be exerted. Statistical heterogeneity will be investigated using $I^{2}$ test. $I^{2} \leq 50 \%$ means little or no statistical heterogeneity across the included trials, and a fixed-effects model will be practiced. $I^{2}>50 \%$ indicates considerable heterogeneity, and a randomeffects model will be utilized. If statistical heterogeneity is minor among included trials, we will undertake meta-analysis based on the similar study and patient characteristics, interventions, controls, and outcome indicators. If statistical heterogeneity is considerable across the eligible studies, we will perform subgroup 
analysis to explore possible sources for such heterogeneity. If it is still not possible to conduct meta-analysis after subgroup analysis, we will report outcome results as a narrative summary.

Subgroup analysis: If data are available, we will carry out subgroup analysis to explore the sources of considerable heterogeneity based on the variations in study and patient characteristics, different types of interventions, controls, and outcomes.

Sensibility analysis: We will conduct a sensitivity analysis to monitor the robustness of the study findings based on the methodological weaknesses and missing data.

Country(ies) involved: China.

Keywords: Femoral head avascular necrosis; total hip arthroplasty; efficacy; complications. 